\title{
Improving the OFDMA performance with common carrier frequency offset correction
}

\author{
Dah-Chung Chang, Yen-Heng Lai \\ Department of Communication Engineering, National Central University, Jhongli City, Taoyuan 320, Taiwan
}

Email address:

dcchang@ce.ncu.edu.tw (D.-C. Chang)

\section{To cite this article:}

Dah-Chung Chang, Yen-Heng Lai. Improving the OFDMA Performance with Common Carrier Frequency Offset Correction. International Journal of Wireless Communications and Mobile Computing. Vol. 2, No. 3, 2014, pp. 35-41. doi: 10.11648/j.wcmc.20140203.11

\begin{abstract}
In an OFDMA system, different carrier frequency offsets (CFOs) are possibly raised due to the mismatch of local oscillators and the Doppler effect because of multiple-antenna channels. In spite of an initial CFO synchronization scheme able to be applied at transmitters, the compensation of residual CFOs is required at the receiver in order to eliminate the inter-carrier interference (ICI) for individual subscribers, especially with an interleaved subcarrier allocation scheme. Unfortunately, conventional ICI mitigation methods cannot simultaneously remove the CFOs caused by multiple subscribers, and therefore, the multiuser interference (MUI) remains. In this case, a common CFO (CCFO) existing among the multiple CFOs is found in relation to the overall OFDMA system performance. In this paper, a CCFO estimation method is proposed at the OFDMA receiver, and the CCFO is then corrected to reach the minimum weighted mean square error (MSE) performance. Numerical results show that new OFDMA receivers after correcting the estimated CCFO significantly improve the overall bit error rate (BER) performance over the conventional receivers.
\end{abstract}

Keywords: OFDMA, Carrier Frequency Offset, Inter-Carrier Interference, Multiuser Interference, Mean Square Error

\section{Introduction}

Orthogonal frequency-division multiplexing (OFDM) is widely used in modern wireless communications for its good ability to reduce the multipath effect. As OFDM is used in a multiple access (MA) system, the combination of the frequency division multiple access (FDMA) method draws a lot of attention to next generations of wireless communications The OFDM multiple access (OFDMA) technology separates groups of OFDM subcarriers allocated to different subscribers for simultaneous uplink transmission from subscriber stations (SS) to a base station (BS). WiMAX and LTE are typical OFDMA systems proposed for the application of wireless metropolitan area networks (MANs) [1]. However, in an OFDMA system, imperfect synchronization due to different carrier frequency offsets (CFOs) at individual transmitting terminals can introduce inter-carrier interference (ICI) among subcarriers and multiple access interference (MAI) among subscribers [2]-[4]. Although some methods can be exploited to initiate the synchronization at transmitters, the CFOs are hard to be completely eliminated since different local oscillators are implemented at the transmitters. Hence, a CFO tracking loop and certain MAI reduction process are usually required at the OFDMA receiver even though some initial synchronization scheme can be employed to reduce the residual CFOs.

Some approaches to dealing with CFOs in an OFDMA system can be found in the literature [5]-[10]. The CFO correction method that is conventionally used in single-user OFDM can be directly applied for different subscribers to cancel the estimated CFOs before the discrete-time Fourier transform (DFT) [5]. But the direct method requires multiple DFT blocks and causes MAI due to different offsets among subscribers. In [6], an alternative method, called the CLJL scheme (abbreviated for the names of the authors), was proposed to compensate for the CFOs effect after the DFT with using circular convolution. Although the CLJL scheme reduces the required number of DFT blocks, the multiuser interference (MUI) components still remain in the compensated results. Huang and Letaief [7] proposed an iterative interference cancellation scheme, called the HL scheme, to reduce the MUI effect. The method proposed in [7] can be regarded as a parallel interference cancellation scheme and the authors showed that only a few of iterations are required to obtain a satisfying performance. Other methods [8][9] considered a 
return path for control information based on maximum likelihood estimation of synchronization parameters.

In previous works [5]-[9], CFOs for different subscribers are estimated and then the correction is performed independently. However, the MUI due to different CFOs still affects the bit error rate (BER) performance even with the use of MUI cancellation schemes [7][10]. It is found that a common CFO (CCFO) existing among the multiple CFOs is related to the overall OFDMA system performance [11][12]. In this paper, we propose a feasible CCFO estimation and correction method in an OFDMA system. As we remove the estimated CCFO before the DFT at the receiver, the overall weighted mean square error (MSE) performance for multiple

subscribers can be minimized such that the average BER performance is improved as well. Simulation results show that the modified CLJL or HL based OFDMA receivers together with the proposed CCFO estimation and correction method have better BER performance than those without employing CCFO correction.

The rest of this paper is organized as follows. Section II introduces the system model and the CLJL/HL methods. The proposed CCFO estimation scheme is also described in this section. Section III contains the simulation results. Section IV concludes this paper.

\section{Proposed CCFO Correction Scheme}

CAS: Carrier Allocation Scheme

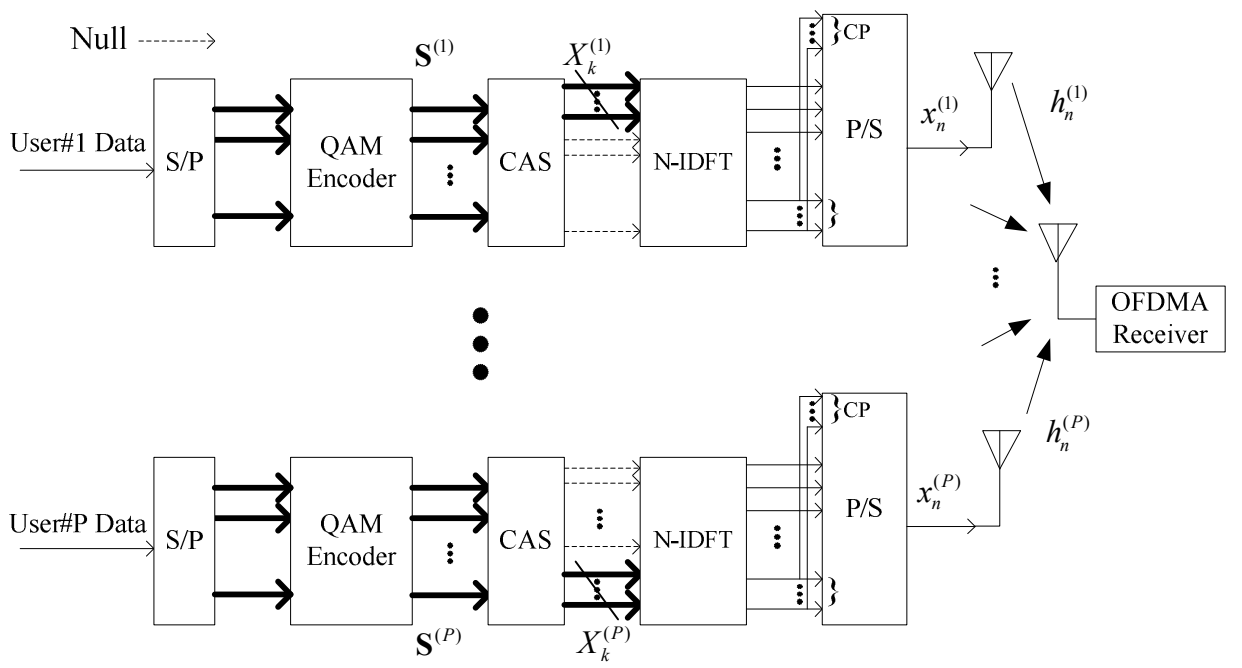

Fig. 1. Structure of the OFDMA Transmitter.

\subsection{System Model and CLJL/HL Methods}

Consider an $N$-point interleaved OFDMA system with $P$ subscribers as depicted in Fig. 1. Each SS communicates with the BS over an independent multipath channel, which is allocated $M$ subcarriers such that $N=M \times P$. The original data symbol for the $m$ th subscriber is denoted by $\mathrm{S}^{(m)}$, $m=1,2, \cdots, P$ and $\mathrm{S}^{(m)}=\left[S_{0}^{(m)} S_{1}^{(m)} \cdots S_{M-1}^{(m)}\right]$. The $M$ signals to be transmitted in the OFDMA system are first mapped into a set of $N$ modulation samples $\left\{X_{k}^{(m)}\right\}, k=0,1, \cdots N-1$, by the scheme of interleaved subcarrier allocation according to

$$
X_{k}^{(m)}=\left\{\begin{array}{lr}
S_{j}^{(m)}, k=j \cdot P+m-1 \\
0, & \text { otherwise }
\end{array}\right.
$$

where $j=0,1, \cdots, M-1$.

Suppose $y_{n}^{(i)}, n=0,1, \ldots, N-1$, is the ideal $i$ th subscriber's symbol after passing through the channel. Let $\varepsilon_{i}$, $i=1,2, \cdots, P$, denote the residual CFO for subscriber $i$ with respect to the $\mathrm{BS}$ and $\varepsilon_{C}$ the $\mathrm{CCFO}$ to be corrected at the $\mathrm{BS}$ before the DFT. The signal after correcting the CCFO at the BS consists of $P$ subscribers' symbols accompanied by corresponding CFO effects and the additive white Gaussian noise (AWGN), which can be given as

$$
r_{n}=\sum_{i=1}^{P} y_{n}^{(i)} e^{j 2 \pi\left(\varepsilon_{i}+\varepsilon_{C}\right) n / N}+z_{n}, n=0,1, \ldots, N-1
$$

where $z_{n}$ is the AWGN. The vector form of the DFT output signal $R_{k}$, where $k$ is the subcarrier index and $0 \leq k \leq N-1$, can be expressed as

$$
\begin{aligned}
\mathrm{R}\left(\varepsilon_{C}\right) & =\operatorname{DFT}_{\mathrm{N}}\left(\mathrm{r}\left(\varepsilon_{C}\right)\right) \\
& =\sum_{i=1}^{P} \mathrm{Y}^{(\mathrm{i})} \otimes \mathrm{C}^{(i)}+\mathrm{Z} \\
& =\mathrm{Y}^{(m)} \otimes \mathrm{C}^{(m)}+\sum_{\substack{i=1 \\
i \neq m}}^{P} \mathrm{Y}^{(i)} \otimes \mathrm{C}^{(i)}+\mathrm{Z}
\end{aligned}
$$

where $\otimes$ denotes the circular convolution, $\mathrm{R}\left(\varepsilon_{C}\right)$ 
$=\left[R_{0}, R_{1}, \ldots, R_{N-1}\right]^{T}, \mathbf{r}\left(\varepsilon_{C}\right)=\left[r_{0}, r_{1}, \ldots, r_{N-1}\right]^{T}$, the $N \times 1$ vector $\mathrm{Y}^{(i)}$ contains the signal for the $i$ th subscriber, $Y_{k}^{(i)}$, with $Y_{k}^{(i)}=\operatorname{DFT}_{\mathrm{N}}\left(y_{n}^{(i)}\right), \mathrm{C}^{(i)}$ is an $N \times 1$ vector containing the value of the equivalent $\mathrm{CFO}$ effects, $C_{k}^{(i)}$, with $C_{k}^{(i)}=\operatorname{DFT}_{\mathrm{N}}\left(e^{j 2 \pi\left(\varepsilon_{i}+\varepsilon_{C}\right) n / N}\right) / N$, and the vector $\mathrm{Z}$ contains the $N$-point DFT results of the AWGN, $Z_{k}$, with $Z_{k}=\operatorname{DFT}_{\mathrm{N}}\left(z_{n}\right) \cdot \operatorname{In}(3)$, the first term is the $m$ th subscriber's received signal and the second term is the MUI due to the CLJL scheme. If the MUI can be ignored and the AWGN power is small compared with the signal power, we can approximate the $m$ th subscriber's received signal as

$$
\begin{aligned}
\hat{\mathrm{R}}^{(m)}\left(\varepsilon_{C}\right) & =\mathrm{Y}^{(m)} \otimes \mathrm{C}^{(m)} \\
& \approx \mathrm{A}^{(\mathrm{m})} \mathrm{R}\left(\varepsilon_{C}\right)
\end{aligned}
$$

where $\mathrm{A}^{(\mathrm{m})}$ is a diagonal matrix with the diagonal elements defined as $\mathrm{A}^{(\mathrm{m})}(i+1, i+1)=1$ for $i \in \Omega_{m}$ and 0 for $i \notin \Omega_{m}$, where $\Omega_{m}$ is the set of subcarriers allocated to the $m$ th subscriber [6][7]. Here, $\mathrm{A}^{(\mathrm{m})}$ acts as a filter that keeps most of the output power for the $m$ th subscriber. From (4), we can restore the $m$ th subscriber's signal $\mathrm{Y}^{(m)}$ from $\hat{\mathrm{R}}^{(m)}\left(\varepsilon_{C}\right)$ by removing the circular convolution operation in the following equation:

$$
\begin{aligned}
\hat{\mathrm{Y}}^{(m)}\left(\varepsilon_{C}\right) & =\mathrm{A}^{(\mathrm{m})}\left(\hat{\mathrm{R}}^{(m)}\left(\varepsilon_{C}\right) \otimes \mathrm{C}^{(m)}\right) \\
& =\mathrm{A}^{(\mathrm{m})}\left(\mathrm{A}^{(\mathrm{m})} \mathrm{R}\left(\varepsilon_{C}\right) \otimes \mathrm{C}^{\prime(m)}\right)
\end{aligned}
$$

where $\mathrm{C}^{\prime(m)}$ denotes the inverse of $\mathrm{C}^{(m)}$, which has components $C_{k}^{\prime(m)}$ and $C_{k}^{\prime(m)}=\operatorname{DFT}_{\mathrm{N}}\left(e^{-j 2 \pi\left(\varepsilon_{m}+\varepsilon_{C}\right) n / N}\right) / N$. The structure of the OFDMA receiver after correcting the CCFO for the CLJL scheme [6] is depicted in Fig. 2.

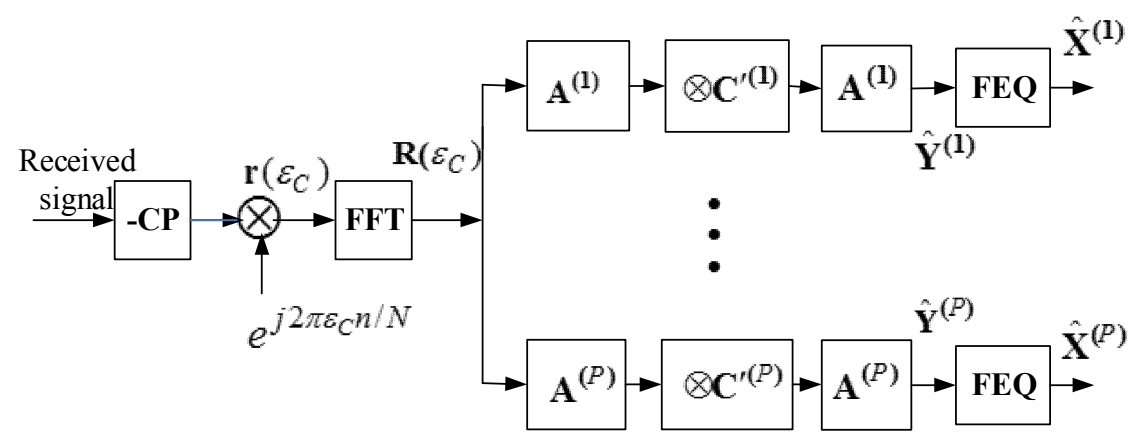

Fig. 2. The OFDMA receiver after correcting the CCFO for the CLJL scheme.

Let $\hat{\mathrm{Y}}^{(i), j}\left(\varepsilon_{C}\right)$ denote the estimate of $\hat{\mathrm{Y}}^{(i)}\left(\varepsilon_{C}\right)$ after performing the $j$ th step of the iterative interference cancellation algorithm proposed in [7]. By ignoring the noise effect, the MUI term can be calculated by

$$
\hat{\mathrm{M}}^{(m), j}\left(\varepsilon_{C}\right)=\sum_{\substack{i=1 \\ i \neq m}}^{P} \hat{\mathrm{Y}}^{(i), j}\left(\varepsilon_{C}\right) \otimes \mathrm{C}^{(i)}
$$

The MUI cancellation algorithm employing the HL scheme can be summarized as follows:

$$
\begin{aligned}
& \text { Initialization: } \text { Set } j=0 \text { and } \\
& \qquad \begin{array}{l}
\hat{\mathrm{Y}}^{(m), j}\left(\varepsilon_{C}\right)=\mathrm{A}^{(m)}\left(\left(\mathrm{A}^{(m)} \mathrm{R}\left(\varepsilon_{C}\right)\right) \otimes \mathrm{C}^{(m)}\right), \\
\quad \text { for } m=1, \ldots, P
\end{array}
\end{aligned}
$$

$$
\begin{aligned}
& \text { Loop: } j=j+1 \\
& \qquad \begin{aligned}
& \text { Set } \overline{\mathrm{Y}}^{(m), j}\left(\varepsilon_{C}\right)=\mathrm{R}\left(\varepsilon_{C}\right)-\hat{\mathrm{M}}^{(m), j}\left(\varepsilon_{C}\right), \\
& \hat{\mathrm{Y}}^{(m), j}\left(\varepsilon_{C}\right)=\mathrm{A}^{(m)}\left(\left(\mathrm{A}^{(m)} \overline{\mathrm{Y}}^{(m), j}\left(\varepsilon_{C}\right)\right) \otimes \mathrm{C}^{(m)}\right), \\
& \text { for } m=1, \ldots, P
\end{aligned}
\end{aligned}
$$

Go back to Loop

\subsection{Effect of CCFO}

Suppose $H_{k}^{(m)}$ is the channel frequency response on the $k$ th subcarrier of the $m$ th subscriber. From (3), after some mathematical manipulation we have

$$
R_{k}\left(\varepsilon_{C}\right)=X_{k}^{(m)} H_{k}^{(m)}+I C I_{k}^{(m)}\left(\varepsilon_{C}\right)+M U I_{k}^{(m)}\left(\varepsilon_{C}\right)+Z_{k}
$$

and 


$$
\begin{aligned}
& I C I_{k}^{(m)}\left(\varepsilon_{C}\right)=\sum_{j \in \Omega_{m}} X_{j}^{(m)} H_{j}^{(m)} D\left(\frac{2 \pi\left(\varepsilon_{m}+\varepsilon_{C}-j+k\right)}{N}\right) \times e^{\frac{j \pi\left(\varepsilon_{m}+\varepsilon_{C}-j+k\right)(N-1)}{N}}-X_{k}^{(m)} H_{k}^{(m)} \\
& M U I_{k}^{(m)}\left(\varepsilon_{C}\right)=\sum_{\substack{i=1 \\
i \neq m}}^{P} \sum_{j \in \Omega_{i}} X_{j}^{(i)} H_{j}^{(i)} D\left(\frac{2 \pi\left(\varepsilon_{i}+\varepsilon_{C}-j+k\right)}{N}\right) \times e^{\frac{j \pi\left(\varepsilon_{i}+\varepsilon_{C}-j+k\right)(N-1)}{N}}
\end{aligned}
$$

where $D(x)=\sin (N x / 2) / N \sin (x / 2)$. From (10) and (11), we can notice that ICI and MUI are influenced by the CCFO.

\subsection{Estimation of CCFO with Minimum Weighted MSE}

From (5) or (8), we can obtain the estimate of the transmitted symbol on the $k$ th subcarrier of the $m$ th subscriber

$$
\hat{X}_{k}^{(m)}\left(\varepsilon_{C}\right)=\hat{Y}_{k}^{(m)}\left(\varepsilon_{C}\right) / H_{k}^{(m)}
$$

We note that $H_{k}^{(m)}$ can be estimated from the preamble or pilots in practical applications. Given the transmitted signal $X_{k}^{(m)}$, we define the weighted MSE as

$$
\xi\left(\varepsilon_{C}\right)=\sum_{m=1}^{P} \sum_{k \in \Omega_{m}} \rho_{m}\left|X_{k}^{(m)}-\hat{X}_{k}^{(m)}\left(\varepsilon_{C}\right)\right|^{2}
$$

where $\rho_{m}$ is the pre-determined weighting coefficient for the $m$ th subscribers, and

$$
\sum_{m=1}^{P} \rho_{m}=1,0 \leq \rho_{m} \leq 1
$$

The purpose of the weighting calculation in (13) is to emphasize the performance for specified subscribers. If all subscribers are equally weighted for the overall performance, we can set $\rho_{m}=1 / P$. In some situations, it may be occurred that in an OFDMA system, not all of allocated subscribers constantly occupy the channel. Hence, the base station can adaptively specify subscribers' performance by changing the weighting coefficients. By the steepest descent approach, the minimization of the weighted MSE can be obtained by calculating the following recursion at time instant $n$ :

$$
\hat{\varepsilon}_{C}(n+1)=\hat{\varepsilon}_{C}(n)-\mu \cdot \nabla_{\varepsilon_{C}} \xi\left(\varepsilon_{C}(n)\right)
$$

where $\mu$ is a step size which controls the convergence rate and the steady-state estimation accuracy. However, it is difficult to obtain the exact formulation for a convergent innovation $\nabla_{\varepsilon_{C}} \xi\left(\varepsilon_{C}(n)\right)$. We approach the derivative by the following numerical method:

$$
\nabla_{\varepsilon_{C}} \xi\left(\varepsilon_{C}(n)\right)=\lim _{\Delta \varepsilon \rightarrow 0} \frac{\xi\left(\varepsilon_{C}(n)+\Delta \varepsilon\right)-\xi\left(\varepsilon_{C}(n)\right)}{\Delta \varepsilon}
$$

where $\Delta \varepsilon$ is chosen as a small value approaching zero, for example, $\Delta \varepsilon=10^{-7}$ is used in our numerical simulation.

It should be noted that $\xi\left(\varepsilon_{C}(n)\right)$ is not a quadratic function of $\varepsilon_{C}(n)$, and hence, $\xi\left(\varepsilon_{C}(n)\right)$ may have some local minimums with respect to $\varepsilon_{C}(n)$. Some routine is required to search for the initial value in the region of global minimum in order to guarantee optimal convergence. A simple method is to divide the whole searching region into several sub-regions for possible initial $\mathrm{CCFO}$ values $\hat{\varepsilon}_{C}(0)$ at first. Then, we can determine the optimal CCFO initial value by finding the minimum value of their MSE metrics from (13). Since this method is proposed for improving performance when coarse synchronization and channel estimation have been achieved by the preamble, pilots or decision feedback data can be used to avoid the requirement of pre-knowing $X_{k}^{(m)}$ shown in (13).

\section{Simulation Results}

We consider an OFDMA system with the DFT size $N=512$, the length of guard-interval is 64 , the number of subscribers is 4 with 128 block subcarriers allocated to each subscriber, eight pilots are used for each subscriber, and the Gray-coded 16-QAM signals are transmitted. The power profile of the impulse response of the multipath channel is assumed to be exponentially decaying with the characteristics $E\left\{|h(n)|^{2}\right\}$ $=\exp (-n / 5), n=0,1, \ldots, 11$. For the purpose of estimating the coarse CFOs and channel responses of the four subscribers, two pilot symbols as the preamble lead in advance of the transmitted data. To avoid the interference among subscribers in using the preambles for initial estimation of CFOs and channel responses [7], the preambles for different subscribers are assigned at non-overlapped symbol slots for simplicity.

We randomly choose two sets of CFO values to see the influence of CCFO correction in this OFDMA system: One is with large $\mathrm{CFO}$ values for the four subscribers and denoted by

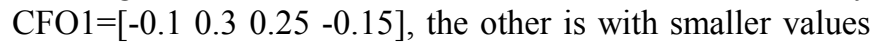

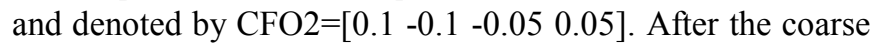
CFOs and channel responses are obtained from preambles, we search for the optimal initial value for CCFO estimation based on the first 30 decision feedback data symbols. Note that during these 30 data symbols, the CCFO effect is temporarily ignored such that the system performance is equivalent to the conventional methods that do not apply CCFO correction. Thereafter, the estimated initial CCFO is used in (15) for recursive estimation. 
In Fig. 3, we show the MSE performance with respect to the $\mathrm{CCFO}$ for the four subscribers in the CFO1 case. That is, for a specified subscriber we assume that its weighting coefficient is unity while the coefficients for others are zero. We compare the performance for the CLJL and HL schemes. It is obvious from the result of the HL scheme that the optimal CCFO value is about to cancelling the CFO effect before the DFT. By adopting the proposed approach for the initial value, we get the

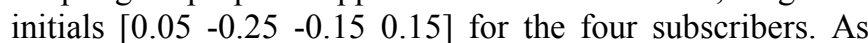
shown in Fig. 4, the proposed CCFO estimation algorithm for the HL scheme finally converges close to the CCFO values with MMSE as shown in Fig. 3. Note that the number of iterations for convergence depends on the choice of the step size. It is a tradeoff between the convergence rate and the steady state performance. A globally optimum $\mathrm{CCFO}$ value can be also found under the consideration of an overall minimum weighted MSE performance, i.e. setting $\rho_{i}=0.25$ for $i=1,2,3$, and 4 . For example, the optimum CCFO value is found about -0.1 for CFO1.
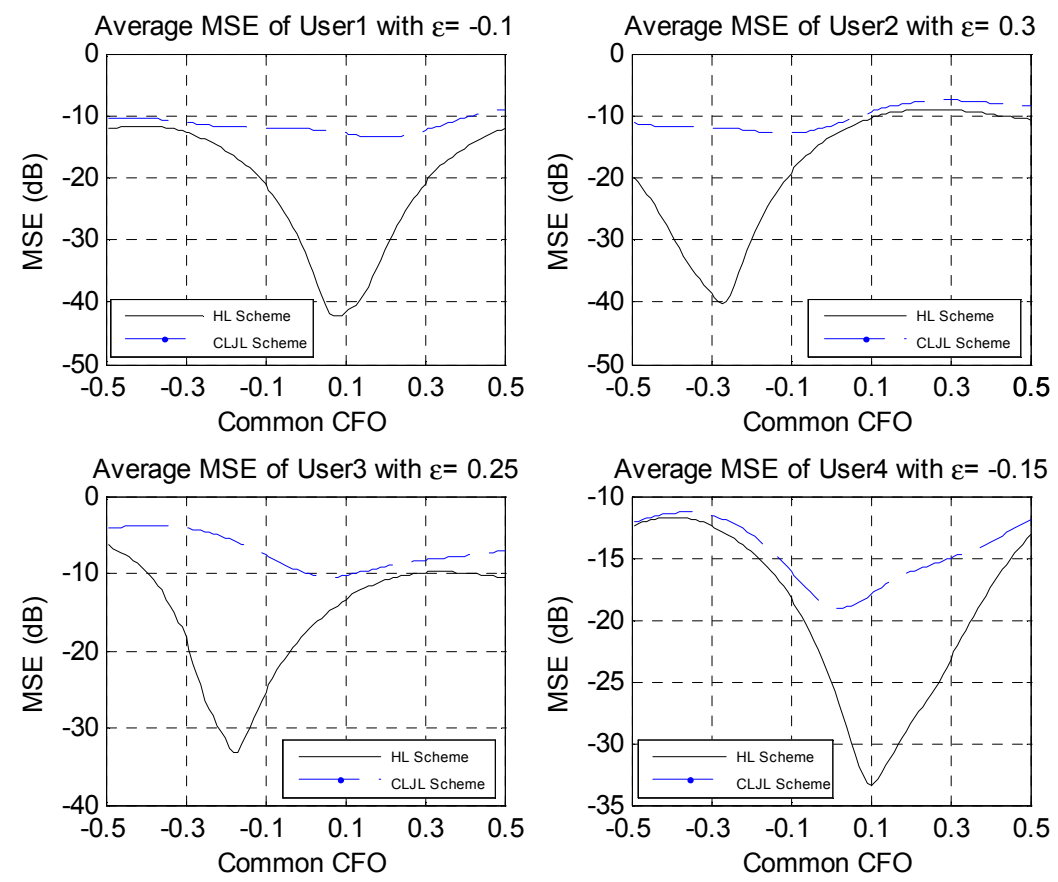

Fig. 3. MSE performance with respect to the CCFO for different subscribers in the CFO1 case.

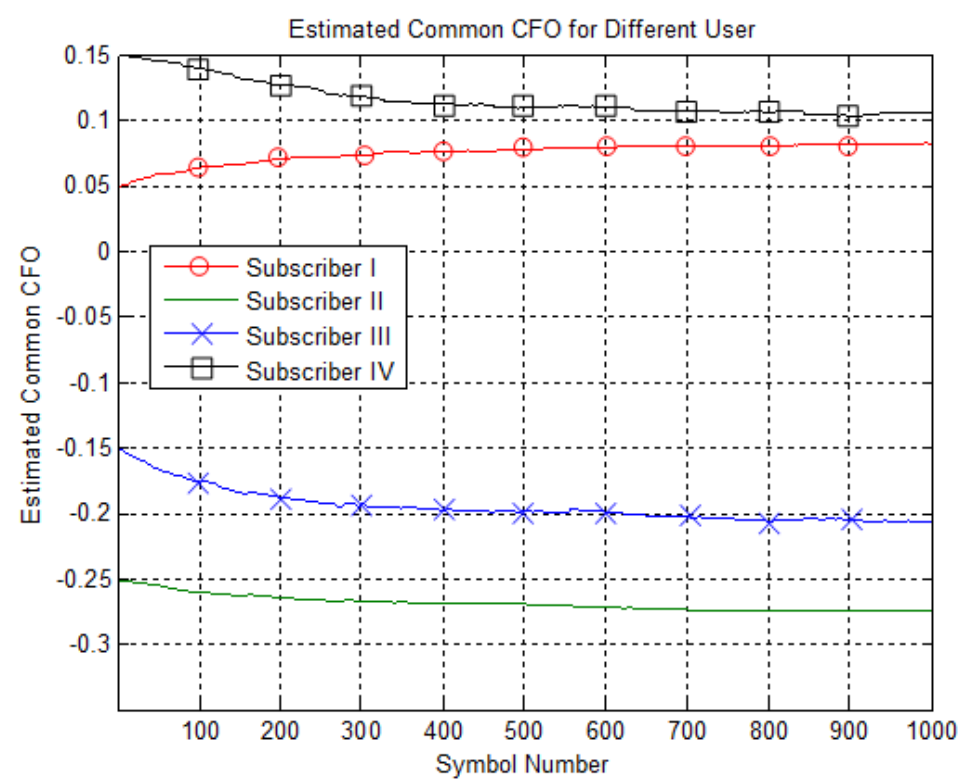

Fig. 4. The learning curves of CCFO estimation for different subscribers. 


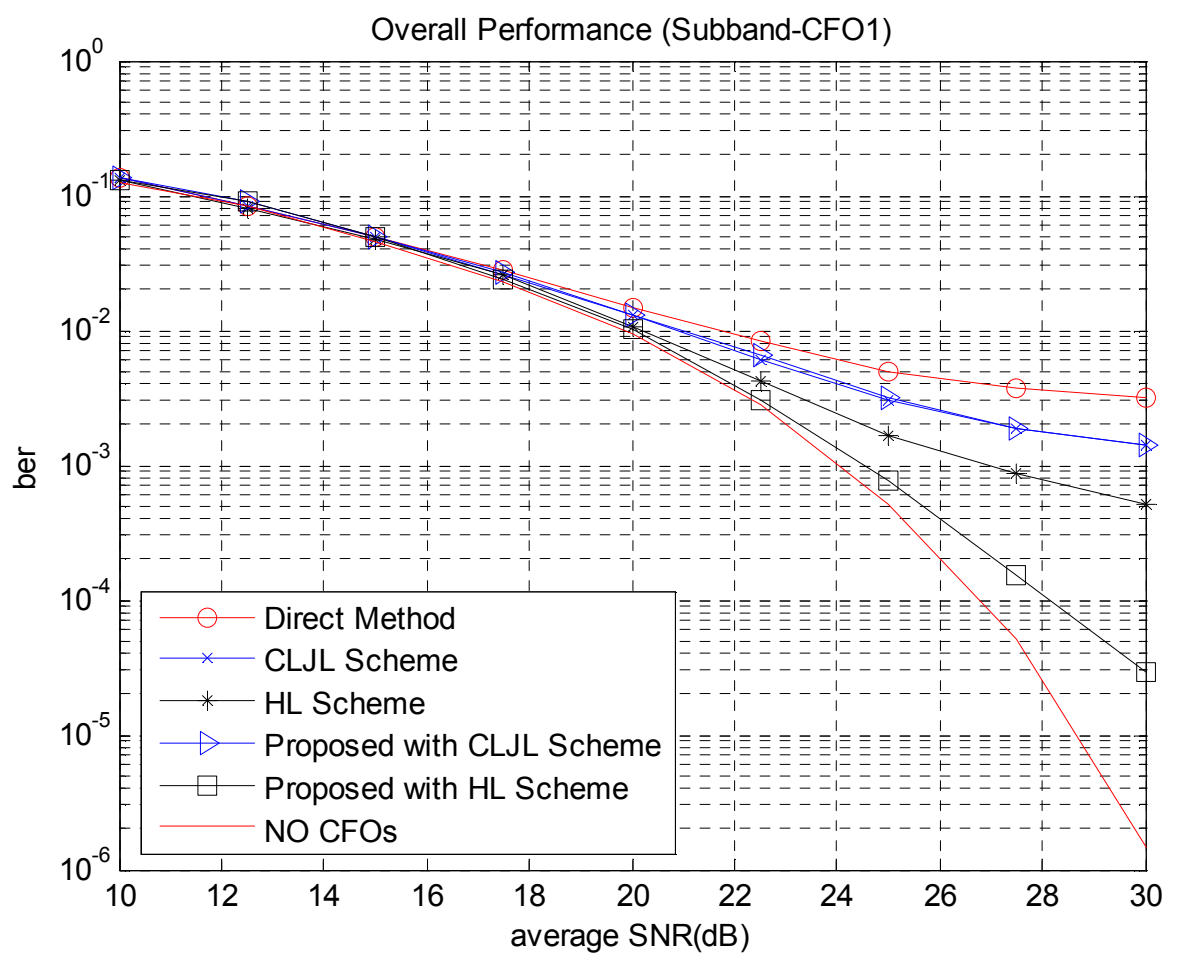

Fig. 5. BER comparison of the overall performance for different CFO compensation methods in OFDMA with CFO1.

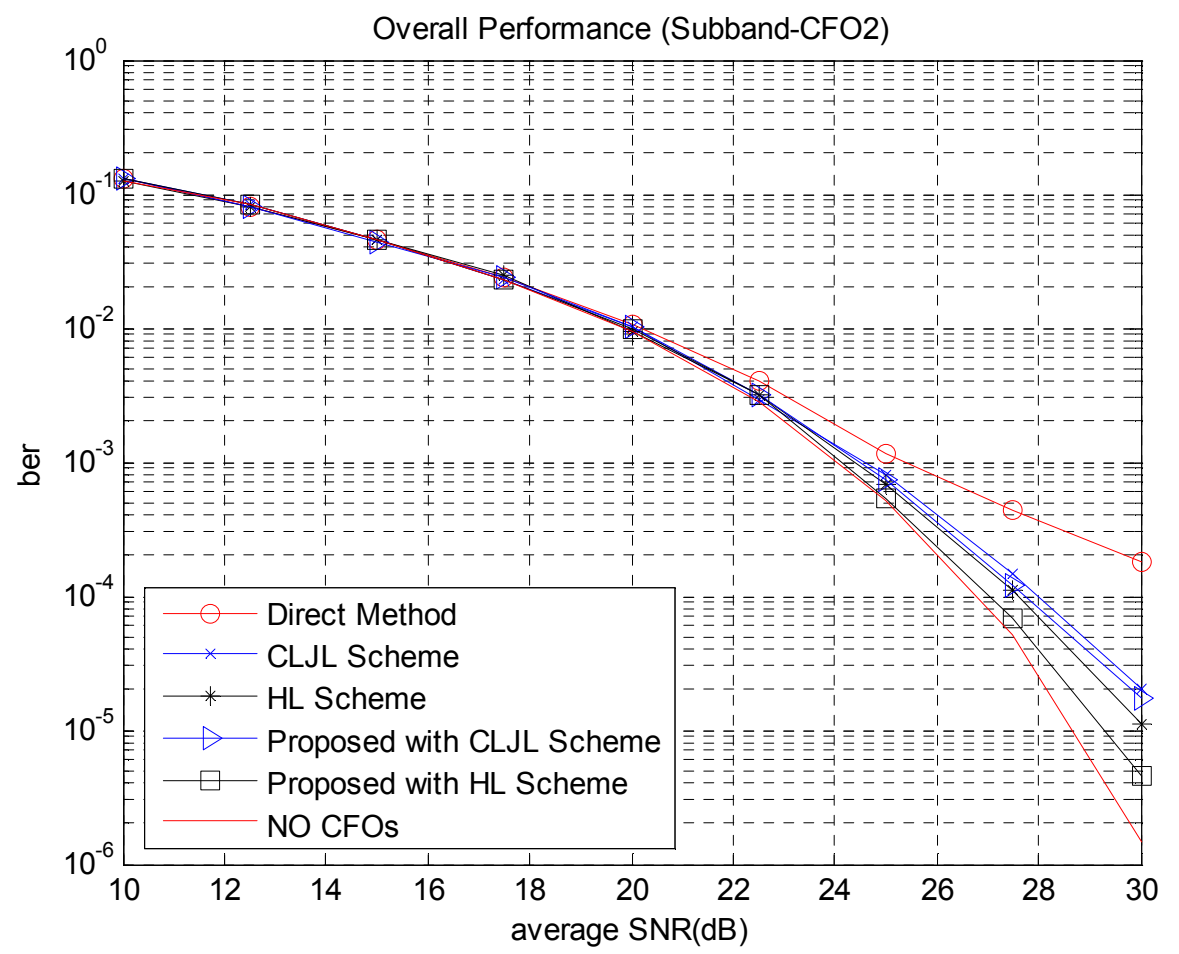

Fig. 6. BER comparison of the overall performance for different CFO compensation methods in OFDMA with CFO2.

We compare the BER performance for different CFOs mitigation schemes in Figs. 5 and 6. The direct method [5] and the CLJL method only compensate for the effect of CFOs, while the MUI effect is not solved. Thus, the HL method that can reduce MUI has better performance than other two methods. However, the proposed CCFO estimation scheme can search for a proper CCFO to minimize the weighted MSE performance. Hence, the proposed method along with the HL's MUI cancellation scheme outperforms others. In Fig. 5, the CFO1 case is simulated to show that a significant performance difference can be noticed. While in Fig. 6 the CFO2 case is simulated, we can observe that the BER performance is also improved with applying the proposed method together with the HL scheme. 


\section{Conclusion}

In conventional methods, the $\mathrm{CFO}$ effect can be compensated for by the CLJL scheme at the BS in the OFDMA system. The MUI effect can be reduced by the HL scheme. In this paper, we show that the MSE performance of the demodulated signals is related to a CCFO which should be corrected in advance of the DFT to reach the optimum weighted MSE. Therefore, we propose a new method for CCFO estimation and correction. From simulation results, a better BER performance can be obtained by applying the CCFO adjustment method along with the CLJL or the HL scheme. Moreover, the performance of the proposed method together with the HL scheme is superior to that of others from our simulation results.

\section{Acknowledgement}

This work was supported in part by the Research Center for Advanced Science and Technology, National Central University as well as the National Science Council of Taiwan under contract NSC 103-2221-E-008-034.

\section{References}

[1] S. Srikanth, P. A. Murugesa Pandian, and X. Femando, "Orthogonal frequency division multiple access in WiMAX and LTE: a comparison," IEEE Commun. Mag., vol. 50, no. 9, pp. 153-161, Set. 2012.

[2] S.-W. Hou and C. C. Ko, "Intercarrier interference suppression for OFDMA uplink in time- and frequency-selective fading channels," IEEE Trans. Veh. Technol., vol. 58, no. 6, pp. 2741-2754, July 2009.

[3] Z. Zhang and C. Tellambura, "The effect of imperfect carrier frequency offset estimation on an OFDMA uplink," IEEE Trans. Commun., vol. 57, no. 4, pp. 1025-1030, Apr. 2009.
[4] T. Yucek and H. Arsian, "Carrier frequency offset compensation with successive cancellation in uplink OFDMA systems," IEEE Trans. Wireless Commun., vol. 6, no. 10, pp. 3546-3551, Oct. 2007.

[5] J.-J. van de Beek et. al, "A time and frequency synchronization scheme for multiuser OFDM," IEEE J. Sel. Areas Commun., vol. 17, no. 11, pp.1900-1913, Nov. 1999.

[6] J. Choi, C. Lee, H. W. Jung, and Y. H. Lee, "Carrier frequency offset compensation for uplink of OFDM-FDMA systems," IEEE Commun. Lett., vol. 4, no. 12, pp. 414-416, Dec. 2000.

[7] D. Huang and K. B. Letaief, "An interference-cancellation scheme for carrier frequency offsets correction in OFDMA systems," IEEE Trans. Commun., vol. 53, no. 7, pp. 1155-1165, July 2005.

[8] M. Morelli, "Timing and frequency synchronization for the uplink of an OFDMA system," IEEE Trans. Commun., vol. 52, no. 2, pp. 296-306, Feb. 2004.

[9] M.-O. Pun, M. Morelli, and C.-C. Jay Kuo, "Maximum-likelihood synchronization and channel estimation for OFDMA uplink transmissions," IEEE Trans. Commun., vol. 54, no. 4, pp. 726-736, Apr. 2006.

[10] S. Manohar, D. Screedhar, V. Tikiya, and A. Chockalingam, "Cancellation of multiuser interference due to carrier frequency offsets in uplink OFDMA," IEEE Trans. Wireless Commun., vol. 6, no. 7, pp.2560-2571, July 2007.

[11] D.-C. Chang, Y.-H. Lai, and Y.-C. Hsu, "Effect of common carrier frequency offset at the OFDMA receiver," in Proceeding of IEEE International symposium on Circuits and Systems (ISCAS), May 2009, pp.201-204.

[12] S. Gaum and R. Kumar, "Performance analysis of OFDMA receiver with common carrier frequency offset (CCFO)," in Proceeding of 2012 World Congress on Information and Communication Technologies (WICT), Oct. 2012, pp. 266-271. 